\title{
The respiratory supercomplex from C. glutamicum
} Agnes Moe ${ }^{1, a}$, Terezia Kovalova ${ }^{1, a}$, Sylwia Króla ${ }^{a}$ David J. Yanofsky ${ }^{b, c}$, Michael Bott ${ }^{d}$, Dan
Sjöstrand ${ }^{\text {a }}$, John L. Rubinstein ${ }^{2, b, c, e}$, Martin Högbom ${ }^{2, a}$ and Peter Brzezinski ${ }^{2, a}$

a Department of Biochemistry and Biophysics, The Arrhenius Laboratories for Natural Sciences, Stockholm University, SE-106 91 Stockholm, Sweden.

${ }^{\mathrm{b}}$ Molecular Medicine program, The Hospital for Sick Children, 686 Bay Street, Toronto, Ontario, Canada M5G 0A4

${ }^{\mathrm{C}}$ Department of Medical Biophysics, The University of Toronto, 101 College Street, Toronto, Ontario, Canada M5G $1 \mathrm{L7}$

${ }^{d}$ Institute of Bio- and Geosciences, IBG-1: Biotechnology, Forschungszentrum Jülich, 52425 Jülich, Germany

e Department of Biochemistry, The University of Toronto, 1 Kings College Circle, Toronto, Ontario, Canada M5S $1 \mathrm{~A} 8$

Keywords: electron transfer, proton transfer, cytochrome $c$ oxidase, cytochrome $b c_{1}$, Bioenergetics, Actinobacteria.

${ }^{1} \mathrm{AM}$ and TK contributed equally to this work.

${ }^{2}$ Corresponding authors: John L. Rubinstein, Martin Högbom and Peter Brzezinski

E-mail: john.rubinstein@utoronto.ca, martin.hogbom@dbb.su.se, peter.brzezinski@dbb.su.se 


\section{Abstract}

Corynebacterium glutamicum is a preferentially aerobic Gram-positive bacterium belonging to the Actinobacteria phylum, which also includes the pathogen Mycobacterium tuberculosis. In the respiratory chain of these bacteria, complexes III (CIII) and IV (CIV) form a $\mathrm{ClII}_{2} \mathrm{CIV}_{2}$ supercomplex that catalyzes oxidation of menaquinol and reduction of dioxygen to water. Electron transfer within the $\mathrm{CIII}_{2} \mathrm{CIV}_{2}$ supercomplex is linked to transmembrane proton translocation, which maintains an electrochemical proton gradient that drives ATP synthesis and transport processes. We isolated the $C$. glutamicum supercomplex and used cryo-EM to determine its structure at $2.9 \AA$ resolution. The structure shows a central $\mathrm{ClII}_{2}$ dimer flanked by a CIV on each side. One menaquinone is bound in each of the $Q_{N}$ and $Q_{P}$ sites in each CIII, near the cytoplasmic and periplasmic sides, respectively. In addition, we identified a menaquinone positioned $\sim 14 \AA$ from heme $b_{\llcorner}$on the periplasmic side. A di-heme cyt. cc subunit provides an electronic connection between each CIII monomer and the adjacent $\mathrm{CIV}$. In $\mathrm{CIII}_{2}$, the Rieske iron-sulfur (FeS) proteins are positioned with the iron near heme $b_{\mathrm{L}}$. Multiple subunits interact to form a convoluted sub-structure at the cytoplasmic side of the supercomplex, which defines a novel path that conducts protons into CIV. 


\section{Introduction}

In the final steps of energy conversion in aerobic organisms, electrons are transferred through the respiratory chain, which consists of membrane-bound proteins that transfer electrons from electron donors, such as $\mathrm{NADH}$, to the final electron acceptor, $\mathrm{O}_{2}$. This electron current drives proton translocation from the negative $(n)$ to the positive $(p)$ side of the membrane, thereby maintaining a voltage difference and a proton concentration gradient that together generate a transmembrane proton motive force (PMF). The free energy stored in the PMF is used by the ATP synthase for production of ATP from ADP and phosphate, or to drive transmembrane transport (1).

NADH dehydrogenases and other enzymes transfer electrons to membrane-soluble quinone $(\mathrm{Q})$, reducing it to quinol $\left(\mathrm{QH}_{2}\right)$. In mitochondria and many bacteria, the reduced $\mathrm{QH}_{2}$ donates electrons to the cyt. $b c_{1}$ complex, also known as complex III, which is found as an obligate dimer $\left(\mathrm{CIII}_{2}\right)$. In each monomer of $\mathrm{CIII}_{2}$ the $\mathrm{QH}_{2}$ binds at the $\mathrm{Q}_{p}$ site, near the $p$ side of the membrane. Energy conservation is realized through a bifurcated electron transfer from $\mathrm{QH}_{2}$, referred to as the $\mathrm{Q}$-cycle (2) (Figure 1). The first electron from $\mathrm{QH}_{2}$ is transferred along the so-called $\mathrm{C}$ branch to a Rieske iron-sulfur protein, which harbors a redox-active $2 \mathrm{Fe}-2 \mathrm{~S}(\mathrm{FeS})$ center. Oxidation of $\mathrm{QH}_{2}$ leads to the release of two protons to the $p$ side of the membrane. The second electron is then transferred along the B branch, passing electrons sequentially to the low-potential heme $b_{\mathrm{L}}$ and the high-potential heme $b_{\mathrm{H}}$ before reducing $\mathrm{Q}$ bound in a second site, the $Q_{N}$ site, located near the $n$ side of the membrane. In canonical $\mathrm{CIII}_{2}$ the FeS center, which is bound in a mobile ectodomain, receives the electron from $\mathrm{QH}_{2}$ while in its $\mathrm{B}$ position in proximity to heme $b_{\mathrm{L}}$. Upon reduction of the FeS center and heme $b_{\mathrm{L}}$, the mobile FeS domain rotates by $\sim 60^{\circ}$ toward the $p$ side to adopt its $C$ position near cyt. $c_{1}$. In the $C$ position the electron from FeS is transferred first to heme $c_{1}$ and then to a water-soluble cyt. c (3) (Figure 1). Cyt. $c$ donates electrons to the last component of the respiratory chain, cytochrome $c$ oxidase (also known as cyt. $a a_{3}$ or complex IV, CIV). After exchange of $\mathrm{Q}$ for $\mathrm{QH}_{2}$ at the $\mathrm{Q}_{\mathrm{p}}$ site of $\mathrm{CIII}$, the sequence of events is repeated, resulting in formation of $\mathrm{QH}_{2}$ at the $\mathrm{Q}_{\mathrm{N}}$ site and abstraction of two protons from the $n$ side of the membrane, contributing further to the PMF (for review, see (4-9)).

The primary electron acceptor from cyt. $c$ in $\mathrm{CIV}$ is a di-nuclear copper $\mathrm{A}$ site, $\mathrm{Cu}_{\mathrm{A}}$, on the $p$ side of the membrane. This copper center transfers electrons to heme $a$ and then to the bi-nuclear catalytic site, composed of a heme $a_{3}$ and copper $\mathrm{Cu}_{\mathrm{B}}$. Upon electron transfer to the catalytic site, heme $a_{3}$ binds an $\mathrm{O}_{2}$ molecule, which is reduced to $\mathrm{H}_{2} \mathrm{O}$, in a process linked 
to proton uptake from the $n$ side of the membrane (Figure 1). The free energy released upon oxidation of cyt. $\mathrm{c}$ and reduction of $\mathrm{O}_{2}$ is conserved by proton pumping from the $n$ to the $p$ side of the membrane (for review, see (10-12)).

Three major CIV families have been defined on the basis of amino-acid sequences as well as functionally important structural features such as proton pathways (13-15). Class A1 members are characterized by a XGHPEVY motif in subunit I and comprises the mitochondrial as well as a large number of bacterial CIVs, including the enzyme from C. glutamicum. In the XGHPEVY motif, $\mathrm{H}$ is a ligand of $\mathrm{CU}_{\mathrm{B}}$ (His265, C. glutamicum CIV numbering) while Y (Tyr269) is covalently linked to His265 (16-19). The A-type CIVs harbor two proton pathways, denoted $\mathrm{D}$ and $\mathrm{K}$, used for proton uptake from the $n$ side of the membrane (16-19). The K pathway transfers two protons to the catalytic site upon reduction of heme $a_{3}$ and $\mathrm{Cu}_{\mathrm{B}}$, while the $\mathrm{D}$ pathway is used for transfer of two protons to the catalytic site after binding of $\mathrm{O}_{2}$ to heme $a_{3}$ and for all protons that are pumped across the membrane (20-24).

The respiratory chains of Gram-positive bacteria of the phylum Actinobacteria do not harbor genes for a water-soluble cyt. c (25) and the series of events that allows a Q-cycle is not as well understood. In these organisms $\mathrm{CIII}_{2}$ and CIV form an obligate $\mathrm{CIII}_{2} \mathrm{CIV}_{2}$ supercomplex in which electron transfer between CIII and CIV is mediated by a di-heme cyt. $c c$ domain that replaces both cyt. $c_{1}$ of the canonical $\mathrm{CIII}_{2}$ and the water-soluble cyt. $c$, as shown e.g. in Mycobacterium smegmatis (26-28) and Corynebacterium glutamicum (29-33) (Figure 1, lower part).

In order to gain insight into electron transfer and proton translocation in the $\mathrm{CIII}_{2} \mathrm{CIV}_{2}$ supercomplex we determined a high-resolution cryo-EM structure of the supercomplex from C. glutamicum. The structure shows density for a menaquinone (MQ) bound in each of the $Q_{N}$ and $Q_{P}$ sites of each CIII monomer. In addition, an MQ was found in a novel site on the membrane $p$ side, $\sim 14 \AA$ from heme $b_{\mathrm{L}}$. As with the $M$. smegmatis supercomplex (26), an extended loop of the QcrB subunit covers the cytoplasmic opening of the D proton pathway of CIV, defining a novel proton-entry route via protonatable residues of QcrB. The FeS ectodomain in $\mathrm{ClII}_{2}$ was found to be locked in the B position, which suggests a Q-cycle mechanism that is gated only by local proton transfer rather than by FeS movement. 


\section{Results and Discussion}

Isolation of the supercomplex

The $750 \mathrm{kDa}$ C. glutamicum $\mathrm{ClII}_{2} \mathrm{ClV}_{2}$ supercomplex was purified using a Strep-tag on the CtaD subunit of CIV (29) (supplementary Figure S1 and Table S1). Absorbance difference spectroscopy suggests a heme $a: b: c$ ratio of approximately $1: 1: 1$, consistent with the $\mathrm{ClII}_{2} \mathrm{CIV}_{2}$ composition of the supercomplex $(29,33)$. Mass spectrometry identified all of the subunits of CIII (QcrA, QcrB, QcrC) and CIV (CtaC, CtaD, CtaE), except for CtaF, which is a hydrophobic membrane protein that is likely difficult to detect by mass spectrometry, as suggested for the equivalent subunit in M. smegmatis (26). Three additional peptides associated with the $\mathrm{CIII}_{2} \mathrm{CIV}_{2}$ supercomplex were identified by mass spectrometry: P20 (later renamed to PRSAF1), P24, and P29 (later renamed to LPqE) (29). Peptide P24 was not identified in the structure. The $\mathrm{MQH}_{2}: \mathrm{O}_{2}$ oxidoreductase activity of the supercomplex was $\sim 100 \mathrm{~s}^{-1}$, consistent with earlier measurements $(29,32)$.

\section{Overall structure of the supercomplex}

To understand the mechanism by which the $C$. glutamicum $\mathrm{II}_{2} \mathrm{IV}_{2}$ supercomplex links electron transfer to proton translocation, we determined its structure by cryo-EM to a nominal resolution of $2.9 \AA$ (supplementary Figures S2-S3 and Table S2). Identifiers for proteins found in the $C$. glutamicum respiratory supercomplex are summarized in Table $\mathbf{S 1}$. The map shows that the core of the supercomplex is composed of a $\mathrm{ClII}_{2}$ dimer flanked by two distal CIV monomers (Figure 2A). This overall arrangement and its geometry is the same as the $M$. smegmatis supercomplex $(26,27)$.The core of CIV is composed of four subunits, CtaC-F (Figure 2B), while each protomer of $\mathrm{ClII}_{2}$ is composed of three subunits, QcrA-C (Figure 2 C) $(25,33)$. An additional six subunits were identified in the $C$. glutamicum supercomplex structure (Figure 2A), two of which (LPqE and PRSAF1) were also found in the M. smegmatis supercomplex structure (27). We propose a new unifying nomenclature and refer to these additional subunits as AscX (Actinobacterial supercomplex, subunit X), except for LpqE (P29), which is an established name (where applicable, the previously-used names are given below in parentheses).

The map allowed for construction of an atomic model for all subunits except for AscD and AscE (supplementary Figure S4 and Table S2). On the periplasmic side of the supercomplex subunit LpqE is attached to the membrane via an N-terminal lipid anchor (Figure 2A, colored in gold). It also interacts with the cyt. cc domain of QcrC, and subunits QcrA, CtaC, and CtaD. As shown previously, LpqE did not co-purify with CIII und CIV alone, suggesting that the interaction with QcrC, which is absent in both single complexes, is 
necessary for the presence of LpqE. Consequently, LpqE may be involved in assembly of the supercomplex (29). Subunit AscA (colored in light salmon) is attached to both $\mathrm{CIII}_{2}$ and $\mathrm{CIV}$. The N-terminal part of the protein starts with a short loop that continues to form two transmembrane $\alpha$-helices attached to subunit QcrB of $\mathrm{ClII}_{2}$. A loop formed by 54 residues near the $C$ terminus of AscA interacts with a loop of CtaD, and the two transmembrane $\alpha$-helices. Like LpqE, AscA was not co-purified with the isolated CIII and CIV complexes, indicating that interaction with both $\mathrm{QcrB}$ and $\mathrm{CtaD}$ is required for co-purification.

A 63-residue stretch of an unknown protein, denoted AscB (colored purple), was identified in the map. A tentative sequence was modeled based on the density and used to search the NCBI (ncbi.nlm.nih.gov) database for potential matches. This search identified protein GenBank: Cg0775, which provides a convincing representation of the complete density (supplementary Figure S4B). No protein from the mass spectrometric analysis provided a plausible match. AscB is folded into a transmembrane $\alpha$-helical hairpin that is attached to CtaD. A short peripheral protein chain composed of 63 amino acid residues, named $\mathrm{AscC}$ (colored in violet) and also conserved in mycobacteria, was also modelled, as described above for AscB, based on GenBank: Cg0935 (supplementary Figure S4B). This protein is partially attached to QcrB on the cytoplasmic side of the supercomplex. The protein chain extends to contact CtaE and CtaF, as well as a QcrB loop that covers one of the proton pathways of CIV (see below). AscD (colored in blue) forms a transmembrane $\alpha$-helical hairpin that is a part of CIV. It is located between the membrane-facing CtaF $\alpha$-helical hairpin and $\operatorname{CtaC} \alpha$-helical hairpin. The sequence of this protein chain is unknown. Its $\mathrm{N}$ - and C-terminal loops are also part of a cytoplasmic side sub-structure, discussed in more detail below. AscE (colored in dark red) is attached to the FeS domain of QcrA and to LpqE. The resolution of this part of the structure is low and it was therefore modelled as polyalanine. The density also shows a similar lipid anchor to that of LpqE.

The structure of the M. smegmatis supercomplex showed a SodC-type Cu-containing superoxide dismutase (SOD) dimer, which is also composed of a lipobox motif attached to a lipoprotein segment $(26,27)$. The $C$. glutamicum strain used in the current study harbors only an Mn-containing SodA (34). AscE of the C. glutamicum supercomplex is located at the equivalent position of the $M$. smegmatis SodC N-terminal anchor (Figure $2 \mathrm{~A}$ and supplementary Figure S5) and no SOD was found attached to the $C$. glutamicum supercomplex. 
Density attributed to putative integral lipid molecules (35) was identified at 61 positions within the supercomplex. Unidentifiable lipids were modeled as hydrocarbon chains (supplementary Figure S6). Cardiolipin (CL), commonly found in membranes that are involved in maintaining an electrochemical proton gradient $(36,37)$, is identified at 14 positions. Four CL molecules are found at the interface of CIII and CIV in each half of the $\mathrm{ClII}_{2} \mathrm{CIV}_{2}$ supercomplex, consistent with a role in supporting supramolecular interactions in respiratory supercomplexes (38). A CL is also found at the monomer-monomer interface of the $\mathrm{CIII}_{2}$ dimer and one $\mathrm{CL}$ is bound to $\mathrm{CtaD}$ facing $\mathrm{AscA}$ and $\mathrm{QcrB}$, further suggesting a role in higher-order assembly of complexes. In addition, another $\mathrm{CL}$ is found in a cavity defined by subunits $\mathrm{CtaE}$ and $\mathrm{CtaF}$ of CIV suggested to be used for $\mathrm{O}_{2}$ diffusion to the catalytic site (39, 40) (see below). All CLs are oriented with their negatively charged headgroups toward the $n$ side of the membrane (see (36)).

\section{Overall structure of Complex III}

The FeS-containing ectodomain on the periplasmic side of QcrA is anchored by three transmembrane $\alpha$-helices (TMH 1-3), one of which (TMH 1) is swapped between CIII monomers in the dimer and occupies the same position where the single transmembrane $\alpha$-helix of the Rieske iron-sulfur protein is found in the canonical CIII (Figure $\mathbf{2 C}$, colored in blue). The two additional transmembrane $\alpha$-helices (TMH2 and 3) from QcrA are formed by an $\sim 80$ residue $\mathrm{N}$-terminal extension not found in canonical $\mathrm{CIII}$. TMH2 occupies the position where the transmembrane $\alpha$-helix of subunit cyt. $c_{1}$ is found canonical CIII, which in the C. glutamicum structure is shifted towards the middle of the supercomplex (see also (26)). The FeS ectodomain of the C. glutamicum CIII is fixed in the B position by the LpqE subunit on the periplasmic side of the protein, which was also noted in one structure of the M. smegmatis supercomplex (27). This tight interface between the QcrA ectodomain and cyt. cc would preclude movement of the ectodomain.

The QcrC subunit is composed of a di-heme cyt. $c c$ (cyt. $c_{\mid}$and $c_{\mid l}$, Figure 1, lower part) head domain and a transmembrane $\alpha$-helix, which is displaced compared to that of cyt. $c_{1}$ subunit in the canonical CIII (Figure 2C, colored in magenta). The C-terminal sequence of QcrC forms a single transmembrane $\alpha$-helix that contacts QcrB. Cyt. $c$ । of the cyt. cc domain interacts with the QcrA ectodomain on the opposite side from the FeS center, while cyt. $c_{\| I}$ is bound near the electron-accepting $\mathrm{Cu}_{\mathrm{A}}$ site of $\mathrm{CIV}$. This arrangement of $c_{\mathrm{C}} \mathrm{c} \mathrm{c}_{1}, \mathrm{cyt}_{\mathrm{t}} \mathrm{c}_{\| 1}$, and $\mathrm{Cu}_{\mathrm{A}}$ provides an electronic connection between $\mathrm{CIII}$ and each $\mathrm{CIV}$ of the supercomplex. 
Subunit QcrB (Figure 2C, colored in green) consists of 8 transmembrane $\alpha$-helices and harbors hemes $b_{\mathrm{L}}$ and $b_{\mathrm{H}}$, which occupy the same positions as in the canonical (41) and M. smegmatis $(26,27) \mathrm{CIII}_{2}$. In addition, the $Q_{p}$ and $Q_{N}$ quinone-binding sites are defined in part by residues of $Q c r B$. The $C$ terminus of $Q c r B$ is extended by 137 residues, not present in the canonical CIII, on the cytoplasmic side of the supercomplex (30). About 20 of these residues form a loop that contacts the CIV subunit CtaD (26).

\section{Quinone binding in complex III}

The $Q_{p}$ site is typically empty in X-ray crystal structures of canonical $\mathrm{CIII}_{2}$ from a wide range of organisms, and was identified from the positions of the inhibitors stigmatellin and myxothiazol in inhibitor-bound structures of the complex (6). A recent cryo-EM study revealed ubiquinone (UQ) bound at the $\mathrm{Q}_{\mathrm{P}}$ site of the $\mathrm{Cl}-\mathrm{CIII}_{2}$ mammalian supercomplex, but only in one monomer of $\mathrm{ClII}_{2}$ (42). The map of $\mathrm{ClII}_{2} \mathrm{ClV}_{2}$ supercomplex reveals density for $\mathrm{MQ}$ adjacent to the FeS cluster in each CIII monomer, thereby defining the $Q_{p}$ site in C. glutamicum (Figure 3A,B and supplementary Figure S7A). This site overlaps with the UQ site identified in mammalian CIII (42) (supplementary Figure S7B). We designate the MQ molecule in this position as MQ1a (Figure 3A-C). In structures of the $M$. smegmatis supercomplex density was seen at a distal position near the entrance to the Qp cavity (26, 27), which we designated as MQ1b (supplementary Figure S7C).

The $Q_{p}$ cavity in the $C$. glutamicum CIII is larger than in the $M$. smegmatis enzyme. The density corresponding to $\mathrm{MQ}$ observed in the $\mathrm{Q}_{\mathrm{P}}$ site of the $C$. glutamicum $\mathrm{CIII}$ is diffuse, spanning across a position equivalent to MQ1b (supplementary Figure S7A). This diffuse density could correspond to a single MQ bound only at MQ1a, or to averaging of two CIII populations in which one MQ bound in either MQ1a or MQ1b. In either case, observation of $\mathrm{MQ}$ at positions MQ1a or MQ1b in C. glutamicum and $M$. smegmatis, respectively, suggests that there are two possible binding modes in or just outside of the $Q_{p}$ site, respectively. Two $\mathrm{QH}_{2}$ binding positions were suggested in a proposed mechanism for canonical $\mathrm{CIII}_{2}$ in which $\mathrm{QH}_{2}$ initially binds in a "stand-by" site and is then re-located into an oxidation site that is formed transiently after docking of the FeS domain in the B position of (7). Furthermore, early EPR data indicated two Q-binding positions in the $Q_{P}$ site of $R$. capsulatus cyt. $b c_{1}$ (43). Two possible MQ binding positions inside and just outside of the Qp cavity is also reminiscent of the two $Q$-binding positions near the $Q_{B}$ site in a crystal structure of a dark-adapted photosynthetic reaction center from Rhodobacter sphaeroides (44). 
Structural studies of the $M$. smegmatis $\mathrm{CIII}_{2} \mathrm{CIV}_{2}$ supercomplex (26) identified density corresponding to MQ in an unexpected location, near the Tyr of the PDFY motif in CIII, which is equivalent to the canonical PEWY Q-binding motif (45). This MQ is at the vertex of a triangle formed with heme $b_{\llcorner}(\sim 20 \AA$ ) and the FeS center ( 20 $\AA$ ) (supplementary

Figure S8A). In the $C$. glutamicum structure the corresponding position in the enzyme cannot accommodate MQ because it is occupied by Trp265 (supplementary Figure S8B). The positions of Trp265 in C. glutamicum and the equivalent Trp276 in M. smegmatis differ, presumably because the former accommodates a smaller Val instead of Phe in its Q-binding motif (PDVY in C. glutamicum) (supplementary Figure S8BC). In C. glutamicum we observed $\mathrm{MQ}$, which we designate MQ2 at a different location at the $p$ side of the membrane, $14 \AA$ from heme $b_{\mathrm{L}}$ and 30-35 $\AA$ from the $Q_{\mathrm{p}}$ site (Figure 3C-E and supplementary Figure S8A). The equivalent location in the $M$. smegmatis structures harbors a lipid tail density $(26,27)$. Identification of a second Q-binding site on the $p$ side of CIII in both C. glutamicum and M. smegmatis suggests a functional role, which is discussed below.

$M Q$ is also found in the $Q_{N}$ site (supplementary Figure $\mathbf{S 9}$ colored in pink), with the head group at the same position seen previously in the canonical (41) and M. smegmatis (26, 27) CIII.

\section{The Q-cycle of complex III}

The bifurcated electron transfer from $Q_{2}$ at the $Q_{p}$ site is fundamental for the $Q$-cycle mechanism that conserves energy in CIII (Figure 1) (46). As outlined in the Introduction section, in this process the first electron from $\mathrm{QH}_{2}$ is transferred to FeS while the second is transferred to heme $b_{\mathrm{L}}$ :

$$
\mathrm{QH}_{2} \mathrm{FeS}_{\mathrm{L}} \stackrel{k_{1}}{\longleftarrow} \mathrm{SQ}_{k_{-1}} \mathrm{FeS}^{-} b_{\mathrm{L}} \stackrel{k_{2}}{\longrightarrow} \mathrm{QFeS}^{-} b_{\mathrm{L}}^{-}
$$

where $\mathrm{SQ}^{\bullet-}$ is a putative semiquinone. In canonical CIII, rotation of the FeS domain from the electron-receiving $B$ position to the electron-donating $C$ position near $c y t . c_{1}$ is thought to be part of the mechanism that allows bifurcated electron transfer $(6,7,9)$. However, this movement of the FeS domain is not required for electron bifurcation (47).

Oxidation of $\mathrm{QH}_{2}$ at the $\mathrm{Q}_{\mathrm{P}}$ site has been studied primarily with the canonical CIII $(7,48$, 49). Upon $\mathrm{QH}_{2}$ binding, the FeS domain moves to the $\mathrm{B}$ position and $\mathrm{QH}_{2}$ forms a hydrogen bond with the FeS ligand His181 (S. cerevisiae numbering), which also receives a proton from $\mathrm{QH}_{2}$ upon electron transfer to FeS (50-54). The second electron is transferred to heme $b_{\mathrm{L}}$, 
along with proton transfer, presumably to Glu272, which is part of the Q-binding PEWY motif in $S$. cerevisiae $(7,49,51)$. The protonated Glu272 is suggested to rotate toward the heme $b_{\mathrm{L}}$ propionate, which transiently binds the proton $(49,51)$ before it is released to the $p$ side aqueous phase. After transfer of the second electron to heme $b_{\mathrm{L}}$ the FeS domain moves to the $C$ position, which allows electron transfer to $c y t$. $c_{1}$ and release of the proton from His181 to the aqueous phase on the $p$ side.

Because the $C$. glutamicum FeS domain is locked in the B position, a mechanism other than movement of the FeS domain must exist to explain the proton-coupled electrontransfer reactions linked to $\mathrm{MQH}_{2}$ oxidation. Similar to the canonical CIII, it is feasible that in the $C$. glutamicum enzyme an electron and a proton are transferred from $\mathrm{MQH}_{2}$ in the $\mathrm{Q}_{\mathrm{p}}$ site to FeS and its His355 ligand (Figure $3 A$ ), respectively (Figure $4, \mathrm{e}_{1}^{-}$and $\mathrm{H}^{+}{ }_{1}$, top left). The equivalent of S. cerevisiae Glu272 in C. glutamicum is Asp295 (Figure 3A) of the PDVY motif, which is located near the heme $b_{\mathrm{L}}$ propionates, but the shorter Asp side chain is too short to reach $\mathrm{MQH}_{2}$ in the Qp site to accept a proton. Instead, Asp302 (Figure 3A), located $\sim 5 \AA$ from $\mathrm{MQH}_{2}$ in the $Q_{p}$ site could receive a proton upon transfer of the second electron from $\mathrm{SQ}^{\bullet-}$ to heme $b_{\mathrm{L}}$ (Figure 4, $\mathrm{e}_{2}^{-}$and $\mathrm{H}^{+}{ }_{2}$, top left). In many Actinobacteria a Glu residue is found at this position, which could also serve as a proton acceptor. Arg306 is 3 A from Asp302

(Figure 3A), which points to a possible route for proton release to the $p$ side aqueous solution (Figure 4, top right). Interestingly, Asp302 is 3 Å from His355 suggesting that this residue is also on a proton-transfer pathway from His355. This architecture offers a plausible mechanism for Q-cycle electron branching in C. glutamicum that is guided by local protontransfer reactions in the protein matrix. We suggest that after the initial oxidation of $\mathrm{MQH}_{2}$ (Figure 4, top left), the electron is stabilized by the His355 proton. Thus, electron transfer from $\mathrm{FeS}^{-}$to heme $c_{1}$ is not possible because His355 cannot be deprotonated until $\mathrm{H}_{2}^{+}$is released from Asp302. If electron transfer from heme $b_{\mathrm{L}}$ to heme $b_{\mathrm{H}}$ occurs as fast as proton transfer from Asp302 to the $p$ side (Figure 4, top right), charge separation along the B branch is accomplished while FeS remains in the reduced state, FeS- The FeS ligand His355 can become deprotonated only after Asp302 loses its proton, which allows electron transfer from FeS ${ }^{-}$to heme $c_{1}$, along the $C$ branch (Figure 4, bottom right). In the final step of the reaction the second proton from D302 is released via R306 (Figure 4, bottom left). This model is supported by the observation that the $\mathrm{FeS}^{-} \rightarrow$ heme $c_{1}$ electron transfer is the slowest of the measured electron-transfer reactions in the $C$. glutamicum supercomplex (32). 
A second $\mathrm{MQ}$ is found at the $p$ side of the supercomplex, $14 \AA$ from heme $b_{\mathrm{L}}$ (MQ2 in Figure 3C-E). The position of MQ2 is different from that observed in the M. smegmatis enzyme $(26,27)$ (supplementary Figure $\mathbf{S 8}$ ). The role of this $M Q$ is unknown, but we speculate that electron transfer via the MQ2 site could provide an alternative electron path that bypasses heme $b_{H}$, thereby decoupling electron transfer through the CIII portion of the supercomplex from generation of a PMF and preventing energy conservation. This pathway would maintain an electron flux through the respiratory chain, for example at low $\mathrm{O}_{2}$ concentrations (1).

\section{Complex IV}

The core subunits of $C$. glutamicum CIV, CtaC and CtaD, are homologous to conserved subunits (SU) II and I, respectively, of canonical CIV. These subunits harbor all redox-active metal cofactors of CIV. Subunit CtaC is composed of two transmembrane $\alpha$-helices and a head domain, which binds the primary electron acceptor, $\mathrm{Cu}_{\mathrm{A}}$. Subunit $\mathrm{CtaD}$ is composed of 12 transmembrane $\alpha$-helices, which bind heme $a$ and form the catalytic site that includes heme $a_{3}$ and $\mathrm{Cu}_{\mathrm{B}}$. The relative positions of the redox-active cofactors within $\mathrm{CtaC}$ and $\mathrm{CtaD}$ are the same in C. glutamicum as in CIV from other organisms (Figure 5A). In canonical CIV the seven transmembrane $\alpha$-helices of SU III form a V-shaped $\mathrm{O}_{2}$ channel $(39,40)$ that harbors three tightly-bound lipid molecules (55). As with the M. smegmatis supercomplex $(26,27)$, SU III from canonical CIV is replaced by two proteins, CtaE and CtaF, in the C. glutamicum enzyme (Figure 2B, brown and yellow) harboring a single CL molecule (supplementary Figure S6). The division of SU III into two parts resembles the supercomplex structure of alternative complex III (ACIII) and CIV in Flavobacterium johnsoniae where the equivalent of SU III has lost the first two transmembrane $\alpha$-helices (equivalent of CtaF) (56).

Previous structures of mammalian, S. cerevisiae, and bacterial CIV revealed a $\mathrm{Mg}^{2+}$ ion $12 \AA$ from $\mathrm{CU}_{\mathrm{B}}$ and $13 \AA$ from the heme $a_{3}$ iron $(18,57-59)$. In addition, a $\mathrm{Na}^{+}$or $\mathrm{Ca}^{2+}$ ion was found to be bound in mitochondrial and bacterial CIV, respectively, at a specific site on the $p$ side of $\operatorname{CIV}(18,58,59)$. In the present structure we found densities at both positions, which we modelled as $\mathrm{Mg}^{2+}$ and $\mathrm{Ca}^{2+}$, respectively (Figure $\mathbf{5 A}$, green and blue spheres, respectively).

The supercomplex structure reveals both the $\mathrm{K}$ and D proton pathways in CIV. The $\mathrm{K}$ proton pathway, used for proton transfer to the catalytic site upon reduction of heme $a_{3}$ and $\mathrm{Cu}_{\mathrm{B}}$, starts near the $n$-side surface at Glu110 (CtaC), and is lined by a number of $\mathrm{CtaD}$ residues, including the conserved central Lys341 as well as Tyr269 at the catalytic site (21, 
$22,60,61)$. The $D$ proton pathway is defined by the highly-conserved Asp116 in the inner part of a cavity in $\mathrm{CtaD}$ and a number of polar residues that span the distance to the highlyconserved Glu267 of the XGHPEVY motif (Figure 5). X-ray crystal structures of CIV from other organisms also revealed about ten water molecules that span the distance between Asp116 and Glu267 (16-19), but these water molecules could not be resolved in the current cryo-EM map. The D pathway is used for proton transfer to the catalytic site as well as for proton pumping to the $p$ side of the membrane after binding of $\mathrm{O}_{2}$ to heme $a_{3}(20,22,60,61)$.

\section{Cytoplasmic side sub-structure}

At the cytoplasmic side of the $C$. glutamicum supercomplex multiple subunits interact to create an intricate sub-structure, which includes secondary structure elements from CtaD, the $\mathrm{C}$ terminus of AscA, a CtaF loop, AscC, and the $\mathrm{N}$ and $\mathrm{C}$ termini of AscD (Figure 5B). The equivalent sub-structure of the $M$. smegmatis supercomplex is composed of fewer components, primarily subunits MSMEG_4692 (Ctal) and MSMEG_4693 (CtaJ) $(26,27)$ that are not present in the $C$. glutamicum supercomplex. Interestingly, as seen in the M. smegmatis supercomplex, the 20-residue QcrB loop (see above) covers the protonuptake cavity around Asp116 (Figure 5B), which in the canonical CIV is exposed to the $n$-side aqueous solution. Conservation of this feature in both the $M$. smegmatis and $C$. glutamicum supercomplexes suggests a functional role. Mutation of the equivalent of Asp116 or residues in its vicinity in other CIVs result in drastic changes in the proton-uptake rate or uncoupling of proton pumping from $\mathrm{O}_{2}$ reduction (62-64). Furthermore, mutation of a Glu residue in a Cterminal flexible loop in $R$. sphaeroides CIV, $10 \AA$ "below" the equivalent of Asp116, near the QcrB extension loop in the $C$. glutamicum structure, resulted in a decrease in the protonpumping stoichiometry by a factor of two (65). Collectively, these data show that the area around the $D$ pathway opening is critical for determining proton-uptake kinetics, presumably by tuning the electrostatic potential thereby providing a proton-collecting antenna (66). In addition, in C. glutamicum a chain of Asp, Glu, and His residues at the C terminus of QcrB provide an alternative proton path from the $n$ side surface to Asp116 (Figure 5B).

We speculate that the structural modification of the cytoplasmic side sub-structure in C. glutamicum is result of differences between the cytoplasmic composition of Gramnegative alphaproteobacteria and mitochondria compared to Gram-positive Actinobacteria, as evident from a higher turgor pressure for the latter (67-69). As outlined above, the proton-collecting antenna around Asp116 determines the proton-uptake kinetics by the D pathway in CIV and any changes to the ionic composition of the cytoplasm are expected to modify this kinetics (66). We propose that the structural modifications around the 
D pathway opening in C. glutamicum optimize the proton-collecting function for the cellular environment of actinobacteria.

\section{Electron transfer from $\mathrm{QH}_{2}$ to $\mathrm{O}_{2}$}

Complexes III and IV are electronically connected by the cyt. cc domain of the QcrC subunit of CIII. In the C. glutamicum structure the distance between FeS and cyt. $c_{1}$ is $21 \AA$ (Figure 5A). Assuming a $\Delta G^{0} \cong+60 \mathrm{meV}$ (33) and a reorganization energy of $0.7 \mathrm{eV}$ yields a time constant for electron transfer from FeS to cyt. $c_{1}$ of $\sim 10 \mathrm{~ms}(70)$, which is consistent with the measured value of $\sim 6.5$ ms for oxidation of the $C$. glutamicum CIII (32). The Fe-Fe distances between hemes $c_{\mid}$and $c_{\| 1}$, and between heme $c_{\|}$and $\mathrm{Cu}_{\mathrm{A}}$ are both $\sim 18 \AA$ (Figure 1) yielding calculated time constants of $\sim 1 \mathrm{~ms}$ for each electron transfer. Kinetic data showed biphasic oxidation of cyt. $c c$ suggesting a time constant for electron transfer to $\mathrm{Cu}_{A}$ in the range $100 \mu \mathrm{s}-2 \mathrm{~ms}(32)$, which is consistent with the estimated value.

\section{Summary}

This study reveals the structure of the respiratory $\mathrm{CIII}_{2} \mathrm{CIV}_{2}$ supercomplex from C. glutamicum. The structure shows $M Q$ bound inside the $Q_{p}$ site cavity and offers insights into how actinobacteria enable energy-conserving Q-cycle electron bifurcation without the mobile FeS domain found in canonical CIII. The structure shows a novel D proton pathway at the opening of CIV where residues from the neighboring QcrB subunit provide a protonentry route from the $n$ side. These findings illustrate the wide variety of structures that allow realization of respiratory pathways in aerobic organisms with particular insight into respiration in actinobacteria such as $M$. tuberculosis where respiration is a validated drug target.

\section{Materials and methods}

\section{Growth of bacteria}

Corynebacterium glutamicum, strain $\Delta C-D_{s t}\left(13032 \Delta c t a D\right.$ with pJC1-cta $\left.D_{s t}\right)$, described before (29), was grown on BHI-Agar plates (33 g/l brain heart infusion broth, $15 \mathrm{~g} / \mathrm{l}$ agar, $20 \mathrm{~g} / \mathrm{l} \mathrm{D}$-(+)-glucose, $25 \mathrm{mg} / \mathrm{l}$ kanamycin). Single colonies were picked, inoculated into $10 \mathrm{ml}$ $\mathrm{BHI}$ culture medium and grown over night using a shaker at $300 \mathrm{rpm}, 30^{\circ} \mathrm{C}$. The pre-culture was diluted into $500 \mathrm{ml} \mathrm{CGXII} \mathrm{medium} \mathrm{(71)} \mathrm{in} \mathrm{a} 2$ I flask and shaken at $160 \mathrm{rpm}, 30^{\circ} \mathrm{C}$ until the $\mathrm{OD}_{600}$ reached 12 . The cells were again diluted into 2 I CGXII medium in a 5 I baffled flask and shaken at $130 \mathrm{rpm}, 30^{\circ} \mathrm{C}$. The cells were harvested at $\mathrm{OD}_{600} 17,10000 \times \mathrm{g}$ for $30 \mathrm{~min}$, JLA 8.1000 rotor (Beckman). 


\section{Membrane preparation}

Cells were homogenized in $4 \mathrm{ml}$ cell lysis buffer $(100 \mathrm{mM}$ Tris- $\mathrm{HCl}$ at pH 7.5, $5 \mathrm{mM}$ $\mathrm{MgSO}_{4}$ ) per gram of cells in the presence of a few crystals of a protease inhibitor phenylmethanesulfonyl fluoride (Sigma) and DNasel (Roche). Cells were broken with a cell disrupter with 4 cycles at $35 \mathrm{kPsi}$ (Constant Systems) and cellular debris was removed by centrifugation at $90000 \times g$ for $20 \mathrm{~min}$ at $4^{\circ} \mathrm{C}$ (45 Ti rotor, Beckman). Membranes were collected by ultracentrifugation at $220000 \times g$ for 90 min at $4^{\circ} \mathrm{C}$ (45Ti rotor, Beckman).

\section{Isolation of supercomplexes}

Membranes were solubilized in $100 \mathrm{mM}$ Tris- $\mathrm{HCl}$ pH 7.5, $100 \mathrm{mM} \mathrm{NaCl}, 2$ mM MgSO 4 , $50 \mathrm{mg} / \mathrm{l}$ avidin (to prevent unspecific binding to the column, see below), 1\% (w/v) DDM to a protein concentration of $5 \mathrm{mg} / \mathrm{ml}$ and incubated for $45 \mathrm{~min}$, at $4^{\circ} \mathrm{C}$ under gentle stirring. Insolubilized material was removed by ultracentrifugation at $39000 \times \mathrm{g}, 20 \mathrm{~min}, 4^{\circ} \mathrm{C}$ (SW41 rotor, Beckman). The supernatant was concentrated with a 100-kDa molecular weight cutoff concentrator (Merck Millipore) until the volume was reduced to $5 \mathrm{ml}$. The concentrated supernatant was then diluted in solubilization buffer without detergent to yield a final DDM concentration of $0.1 \%(\mathrm{w} / \mathrm{v})$ and concentrated again to reach a volume of $<10 \mathrm{ml}$. The concentrated supernatant was applied to a gravity flow Strep-Tactin Superflow column (4 ml bed volume, Iba Lifescience). The column was washed 3 times with 0.5 column volumes of washing buffer (100 mM Tris- $\mathrm{HCl} \mathrm{pH} \mathrm{7.5,} 100 \mathrm{mM} \mathrm{NaCl}, 2 \mathrm{mM} \mathrm{MgSO}_{4}, 0.05 \%$ (w/v) DDM). The protein was then eluted with 3 column volumes of elution buffer $(100 \mathrm{mM}$ Tris- $\mathrm{HCl}$ $\mathrm{pH} 7.5,100 \mathrm{mM} \mathrm{NaCl}^{2} 2 \mathrm{mM} \mathrm{MgSO}_{4}, 0.05 \%$ (w/v) DDM, $2.5 \mathrm{mM}$ D-desthiobiotin). The eluted supercomplex solution was concentrated as described above and further purified by size exclusion chromatography on a Superose 6 Increase 10/300 GL column (GE Healthcare), preequilibrated with buffer (100 mM Tris- $\mathrm{HCl}$ pH 7.5, 100 mM NaCl, 2 mM MgSO $4,0.05$ \% DDM) using an Äkta Pure M25 chromatography system (GE Healthcare) operated at $4^{\circ} \mathrm{C}$ with UV detection at $280 \mathrm{~nm}$ and $415 \mathrm{~nm}$. Collected fractions containing supercomplex were concentrated for further analysis.

\section{Spectral analysis}

The purified supercomplex was analyzed by UV-visible absorption spectroscopy (Cary 100 Spectrophotometer, Agilent Technologies). Difference spectra of the dithionitereduced and oxidized states of the supercomplex were recorded. The reduced - oxidized difference absorption coefficients used to estimate the cofactor stoichiometry were: $\varepsilon_{605-}$ $630=24 \mathrm{mM}^{-1} \mathrm{~cm}^{-1}$ (cyt. $a a_{3}$ ), $\varepsilon_{562-577}=22 \mathrm{mM}^{-1} \mathrm{~cm}^{-1}$ (cyt. $b$ ), and $\varepsilon_{552-540}=19 \mathrm{mM}^{-1} \mathrm{~cm}^{-1}$ (cyt. c) $(28,29)$. 
Preparation of menaquinol

2,3-dimethyl-[1,4]naphthoquinone $(1.8 \mathrm{mg})$ was dissolved in $0.5 \mathrm{ml}$ ethanol to yield a $20 \mathrm{mM}$ solution. Several crystals of sodium borohydride $\left(\mathrm{NaBH}_{4}\right)$ were added to reduce the quinone to quinol. The solution was kept on ice until transparent and $\mathrm{HCl}$ was added until formation of small bubbles in the solution ended. The sample was centrifuged for $10 \mathrm{~min}$ at $10000 \times g$ and the supernatant containing reduced quinol was aliquoted, flash frozen, and stored in $-80^{\circ} \mathrm{C}$ until use.

\section{Activity assays}

The $\mathrm{O}_{2}$ reduction rate was measured at $25^{\circ} \mathrm{C}$ using a Clark-type oxygen electrode in a buffer containing 100 mM Tris- $\mathrm{HCl}$ pH 7.5, 100 mM NaCl, 2 mM MgSO, $0.05 \%$ DDM. The reaction was initiated by addition of $5 \mu \mathrm{l}$ of $20 \mathrm{mM}$ 2,3-dimethyl-[1,4]naphthoquinol (Rare Chemicals $\mathrm{GmbH}$ ) solution into a $1 \mathrm{ml}$ chamber containing the supercomplex solution $(40 \mathrm{nM})$. The activity was obtained from the initial slope of the graph. The background $\mathrm{O}_{2-}$ reduction rate was measured and subtracted from the $\mathrm{O}_{2}$-reduction rate obtained in the presence of the supercomplex.

\section{Gel electrophoresis}

Blue Native (BN) PAGE was performed according to the manufacturer's instruction with pre-cast gel, NativePAGE ${ }^{\mathrm{TM}} 4-16 \%$ Bis-Tris (Thermo Fisher Scientific). The gel was run at $4^{\circ} \mathrm{C}$ for $60 \mathrm{~min}$ at $150 \mathrm{~V}$, then the cathode buffer was exchanged to anode buffer and run for an additional $40 \mathrm{~min}$ at $250 \mathrm{~V}$. The gel was then stained with Coomassie Brilliant Blue. The band corresponding to supercomplex from BN PAGE was subjected to mass spectrometry, which indicated the presence of both complexes $\mathrm{II}_{2}$ and IV, as well as two additional subunits, LpqE and AscA (P20, PRSAF1). AscB and AscC were not identified in the mass spectrometric analysis.

SDS PAGE was performed according to manufacturer's instruction with pre-cast gel, NuPAGE ${ }^{T M}$ 4-12\% Bis-Tris (Thermo Fisher Scientific) in MES running buffer. Samples were heated at $65^{\circ} \mathrm{C}$ for $30 \mathrm{~min}$ and run at $4^{\circ} \mathrm{C}$ for $45 \mathrm{~min}$ at $200 \mathrm{~V}$. The gel was then stained with Coomassie Brilliant Blue.

\section{Grid preparation and cryo-electron microscopy}

Purified supercomplexes $(3 \mu \mathrm{l})$ at a concentration of $10 \mathrm{mg} \mathrm{ml}^{-1}$ was applied to holey carbon film coated copper EM grids (C flat 2/2 3C T50) that had been glow-discharged in air for $120 \mathrm{~s}$ at $20 \mathrm{~mA}$ (PELCO easiGlow). Grids were blotted for $3 \mathrm{~s}$ at $4^{\circ} \mathrm{C}$ and $100 \%$ humidity before being plunge-frozen in liquid ethane with a Vitrobot Mark IV (Thermo Fisher 
Scientific). Cryo-EM images were collected at $300 \mathrm{kV}$ with a Titan Krios electron microscope (Thermo Fisher Scientific) equipped with a Gatan K2-summit direct electron detector and a Bio-quantum energy filter (Gatan). Data were collected with a nominal magnification of $130000 \mathrm{x}$, corresponding to a calibrated pixel size of $1.06 \AA$. Automated data collection was done with the EPU software package (Thermo Fisher Scientific). A dataset of 2768 movies was collected, each consisting of 40 exposure fractions. The camera exposure rate and the total exposure of the specimen were $7.7 \mathrm{e}$ /pixel/s and $55 \mathrm{e}^{-} / \AA^{2}$, respectively (Table S2).

\section{Image analysis}

All image analysis was performed with cryoSPARC v2 unless otherwise stated (72). Movies were aligned with MotionCor2 (73) and contrast transfer function (CTF) parameters were estimated in patches. Templates for particle selection were generated by $2 \mathrm{D}$ classification of manually selected particle images. A total of 560 000 particle images were selected, images were corrected for local motion (74) and extracted in $310 \times 310$ pixel boxes. The dataset was first cleaned with 2D classification and then with three rounds of $a b$ initio 3D classification and heterogeneous refinement reducing the size to 65000 particle images. Local and global CTF refinement followed by homogeneous refinement without the application of symmetry resulted in a map at $2.9 \AA$ resolution.

Figures were prepared using the software PyMOL (Molecular Graphics System, Version 2.0 Schrödinger, LLC., (75)) as well as UCSF Chimera, developed by the Resource for Biocomputing, Visualization, and Informatics at the University of California, San Francisco, with support from NIH P41-GM103311.

\section{Model building and refinement}

An initial model of all the subunits of the $C$. glutamicum supercomplex was built manually into the $\mathrm{C} 1$ symmetry density map with $2.9 \AA$ A resolution using Coot (76). Subunits $A s c B, A s c C$ and $A s c D$ were initially build as a poly-alanine chains. Sequences of $A s c B$ and AscC were then built into the density map where high enough resolution allowed identification of amino-acid side chains, based on known sequences of the $C$. glutamicum genome using BLAST (77). No sequence match was found for AscD; it remained modelled as a poly-alanine chain. The model was refined using combination of phenix_real_space_refine (78) and manual adjustments in Coot. 


\section{Data deposition}

Data deposition: all electron cryomicroscopy maps described in this article have been deposited in the Electron Microscopy Data Bank (EMDB) (accession nos. EMD-XXXX to EMD$X X X X)$.

\section{Acknowledgements}

We thank Mikael Oliveberg for valuable discussions. This work was supported by the Knut and Wallenberg Foundation (MH, PB), the Swedish Research Council (MH, PB), and Canadian Institutes of Health Research grant PJT162186 (JLR). JLR was supported by the Canada Research Chairs program. Cryo-EM data was collected at the Swedish National CryoEM Facility funded by the Knut and Alice Wallenberg, Family Erling Persson and Kempe Foundations, SciLifeLab, Stockholm University and Umeå University. Mass spectrometry was done at the Mass Spectrometry-based Proteomics Facility at Uppsala University.

\section{References}

1. E. Zelle et al., An energetic profile of Corynebacterium glutamicum underpinned by measured biomass yield on ATP. Metabolic Engineering 65, 66-78 (2021).

2. P. Mitchell, Possible molecular mechanisms of the protonmotive function of cytochrome systems. J. Theor. Biol. 62, 327-367 (1976).

3. Z. Zhang et al., Electron transfer by domain movement in cytochrome bc1. Nature 392, 677-684 (1998).

4. M. Sarewicz et al., Catalytic reactions and energy conservation in the cytochrome bc1 and b6f complexes of energy-transducing membranes. Chem. Rev. 121, 2020-2108 (2021).

5. A. R. Crofts (2004) The cytochrome bc1 complex: Function in the context of structure. in Annual Review of Physiology, pp 689-733.

6. M. Sarewicz, A. Osyczka, Electronic connection between the quinone and cytochrome $c$ redox pools and its role in regulation of mitochondrial electron transport and redox signaling. Physiological Reviews 95, 219-243 (2015).

7. A. Y. Mulkidjanian, Ubiquinol oxidation in the cytochrome bc1 complex: Reaction mechanism and prevention of short-circuiting. Biochimica et Biophysica Acta Bioenergetics 1709, 5-34 (2005).

8. W. A. Cramer, S. S. Hasan, E. Yamashita, The Q cycle of cytochrome bc complexes: A structure perspective. Biochimica et Biophysica Acta (BBA) - Bioenergetics 1807, 788802 (2011).

9. E. A. Berry, H. De Bari, L. S. Huang, Unanswered questions about the structure of cytochrome bc1 complexes. Biochimica et Biophysica Acta - Bioenergetics 1827, 1258-1277 (2013).

10. M. Wikström, V. Sharma, V. R. I. Kaila, J. P. Hosler, G. Hummer, New perspectives on proton pumping in cellular respiration. Chem. Rev. 115, 2196-2221 (2015).

11. P. Brzezinski, R. B. Gennis, Cytochrome $c$ oxidase: exciting progress and remaining mysteries. J. Bioenerg. Biomembr. 40, 521-531 (2008). 
12. J. P. Hosler, S. Ferguson-Miller, D. A. Mills, Energy transduction: Proton transfer through the respiratory complexes. Annual Review of Biochemistry 75, 165-187 (2006).

13. M. M. Pereira, M. Santana, M. Teixeira, A novel scenario for the evolution of haemcopper oxygen reductases. Biochim. Biophys. Acta-Bioenerg. 1505, 185-208 (2001).

14. J. Hemp, R. B. Gennis, Diversity of the heme-copper superfamily in archaea: insights from genomics and structural modeling. Results Probl Cell Differ 45, 1-31 (2008).

15. H. J. Lee, J. Reimann, Y. Huang, P. Ädelroth, Functional proton transfer pathways in the heme-copper oxidase superfamily. Biochimica et Biophysica Acta-Bioenergetics 1817, 537-544 (2012).

16. S. Iwata, C. Ostermeier, B. Ludwig, H. Michel, Structure at $2.8 \AA$ A resolution of cytochrome c oxidase from Paracoccus denitrificans. Nature 376, 660-669 (1995).

17. M. Svensson-Ek et al., The X-ray crystal structures of wild-type and EQ(I-286) mutant cytochrome c oxidases from Rhodobacter sphaeroides. J Mol Biol 321, 329-339 (2002).

18. T. Tsukihara et al., The whole structure of the 13-subunit oxidized cytochrome c oxidase at $2.8 \AA$ A Science 272, 1136-1144 (1996).

19. L. Qin, C. Hiser, A. Mulichak, R. M. Garavito, S. Ferguson-Miller, Identification of conserved lipid/detergent-binding sites in a high-resolution structure of the membrane protein cytochrome c oxidase. Proc Natl Acad Sci U S A 103, 16117-16122 (2006).

20. P. Ädelroth, M. Svensson Ek, D. M. Mitchell, R. B. Gennis, P. Brzezinski, Glutamate 286 in cytochrome aa3 from Rhodobacter sphaeroides is involved in proton uptake during the reaction of the fully-reduced enzyme with dioxygen. Biochemistry 36, 13824-13829 (1997).

21. P. Ädelroth, R. B. Gennis, P. Brzezinski, Role of the pathway through K(I-362) in proton transfer in cytochrome c oxidase from R. sphaeroides. Biochemistry 37, 24702476 (1998).

22. A. A. Konstantinov, S. Siletsky, D. Mitchell, A. Kaulen, R. B. Gennis, The roles of the two proton input channels in cytochrome coxidase from Rhodobacter sphaeroides probed by the effects of site-directed mutations on time-resolved electrogenic intraprotein proton transfer. Proc. Natl. Acad. Sci. U. S. A. 94, 9085-9090 (1997).

23. P. Brzezinski, P. Ädelroth, Pathways of proton transfer in cytochrome c oxidase. J. Bioenerg. Biomembr. 30, 99-107 (1998).

24. V. Sharma, M. Wikström, The role of the K-channel and the active-site tyrosine in the catalytic mechanism of cytochrome c oxidase. Biochimica et Biophysica Acta Bioenergetics 1857, 1111-1115 (2016).

25. M. Bott, A. Niebisch, The respiratory chain of Corynebacterium glutamicum. Journal of Biotechnology 104, 129-153 (2003).

26. B. Wiseman et al., Structure of a functional obligate complex III2IV2 respiratory supercomplex from Mycobacterium smegmatis. Nature Structural and Molecular Biology 25, 1128-1136 (2018).

27. H. Gong et al., An electron transfer path connects subunits of a mycobacterial respiratory supercomplex. Science 362 (2018).

28. J. A. Megehee, J. P. Hosler, M. D. Lundrigan, Evidence for a cytochrome bcc-aa3 interaction in the respiratory chain of Mycobacterium smegmatis. Microbiology 152, 823-829 (2006). 
29. A. Niebisch, M. Bott, Purification of a cytochrome bc1-aa3 supercomplex with quinol oxidase activity from Corynebacterium glutamicum: Identification of a fourth subunit of cytochrome aa3 oxidase and mutational analysis of diheme cytochrome c1. J. Biol. Chem. 278, 4339-4346 (2003).

30. A. Niebisch, M. Bott, Molecular analysis of the cytochrome bc1-aa3 branch of the Corynebacterium glutamicum respiratory chain containing an unusual diheme cytochrome c1. Archives of Microbiology 175, 282-294 (2001).

31. N. Sone et al., A novel hydrophobic diheme c-type cytochrome. Purification from Corynebacterium glutamicum and analysis of the QcrCBA operon encoding three subunit proteins of a putative cytochrome reductase complex. Biochimica et Biophysica Acta - Bioenergetics 1503, 279-290 (2001).

32. S. Graf et al., Rapid Electron Transfer within the III-IV Supercomplex in Corynebacterium glutamicum. Scientific Reports 6 (2016).

33. W. C. Kao et al., The obligate respiratory supercomplex from Actinobacteria. Biochimica et Biophysica Acta - Bioenergetics 1857, 1705-1714 (2016).

34. H. M. El Shafey, S. Ghanem, M. Merkamm, A. Guyonvarch, Corynebacterium glutamicum superoxide dismutase is a manganese-strict non-cambialistic enzyme in vitro. Microbiological Research 163, 80-86 (2008).

35. H. Palsdottir, C. Hunte, Lipids in membrane protein structures. Biochimica et Biophysica Acta - Biomembranes 1666, 2-18 (2004).

36. V. E. Kagan, C. T. Chu, Y. Y. Tyurina, A. Cheikhi, H. Bayir, Cardiolipin asymmetry, oxidation and signaling. Chemistry and Physics of Lipids 179, 64-69 (2014).

37. G. Paradies, V. Paradies, V. De Benedictis, F. M. Ruggiero, G. Petrosillo, Functional role of cardiolipin in mitochondrial bioenergetics. Biochimica et Biophysica Acta Bioenergetics 1837, 408-417 (2014).

38. K. Pfeiffer et al., Cardiolipin Stabilizes Respiratory Chain Supercomplexes. J. Biol. Chem. 278, 52873-52880 (2003).

39. I. Hofacker, K. Schulten, Oxygen and proton pathways in cytochrome c oxidase. Proteins 30, 100-107 (1998).

40. M. Wikström, K. Krab, V. Sharma, Oxygen Activation and Energy Conservation by Cytochrome c Oxidase. Chem. Rev. 118, 2469-2490 (2018).

41. C. Hunte, J. Koepke, C. Lange, T. Roßmanith, H. Michel, Structure at 2.3 Å resolution of the cytochrome bc1 complex from the yeast Saccharomyces cerevisiae cocrystallized with an antibody Fv fragment. Structure 8, 669-684 (2000).

42. J. A. Letts, K. Fiedorczuk, G. Degliesposti, M. Skehel, L. A. Sazanov, Structures of Respiratory Supercomplex I+III2 Reveal Functional and Conformational Crosstalk. Molecular Cell 75, 1131-1146.e1136 (2019).

43. H. Ding, D. E. Robertson, P. L. Dutton, F. Daldal, Cytochrome bc1 Complex [2Fe-2S] Cluster and Its Interaction with Ubiquinone and Ubihydroquinone at the Qo Site: A Double-Occupancy Qo Site Model. Biochemistry 31, 3144-3158 (1992).

44. M. H. B. Stowell et al., Light-Induced Structural Changes in Photosynthetic Reaction Center: Implications for Mechanism of Electron-Proton Transfer. Science 276, 812 (1997).

45. W. C. Kao, C. Hunte, The molecular evolution of the Qo Motif. Genome Biology and Evolution 6, 1894-1910 (2014).

46. A. Osyczka, C. C. Moser, P. L. Dutton, Fixing the Q cycle. Trends in Biochemical Sciences 30, 176-182 (2005). 
47. S. Jünemann, P. Heathcote, P. R. Rich, On the mechanism of quinol oxidation in the bc1 complex. J. Biol. Chem. 273, 21603-21607 (1998).

48. E. A. Berry, L. S. Huang, Observations concerning the quinol oxidation site of the cytochrome bc 1 complex. FEBS Lett. 555, 13-20 (2003).

49. C. Hunte, H. Palsdottir, B. L. Trumpower, Protonmotive pathways and mechanisms in the cytochrome bc 1 complex. FEBS Lett. 545, 39-46 (2003).

50. M. Iwaki et al., Direct Observation of Redox-Linked Histidine Protonation Changes in the Iron-Sulfur Protein of the Cytochrome bc1 Complex by ATR-FTIR Spectroscopy. Biochemistry 44, 4230-4237 (2005).

51. A. R. Crofts et al., Pathways for proton release during ubihydroquinone oxidation by the bc1 complex. Proceedings of the National Academy of Sciences 96, 10021 (1999).

52. U. Brandt, J. G. Okun, Role of deprotonation events in ubihydroquinone:cytochrome c oxidoreductase from bovine heart and yeast mitochondria. Biochemistry 36, 1123411240 (1997).

53. A. R. Crofts et al., Pathways for proton release during ubihydroquinone oxidation by the bc(1) complex. Proc. Natl. Acad. Sci. U. S. A. 96, 10021-10026 (1999).

54. P. R. Rich, The quinone chemistry of bc complexes. Biochimica et Biophysica Acta (BBA) - Bioenergetics 1658, 165-171 (2004).

55. L. Qin, M. A. Sharpe, R. M. Garavito, S. Ferguson-Miller, Conserved lipid-binding sites in membrane proteins: a focus on cytochrome c oxidase. Current Opinion in Structural Biology 17, 444-450 (2007).

56. C. Sun et al., Structure of the alternative complex III in a supercomplex with cytochrome oxidase. Nature 557, 123-126 (2018).

57. J. P. Hosler, M. P. Espe, Y. Zhen, G. T. Babcock, S. Ferguson-Miller, Analysis of sitedirected mutants locates a non-redox-active metal near the active site of cytochrome c oxidase of Rhodobacter sphaeroides. Biochemistry 34, 7586-7592 (1995).

58. C. Ostermeier, A. Harrenga, U. Ermler, H. Michel, Structure at 2.7 A resolution of the Paracoccus denitrificans two- subunit cytochrome $c$ oxidase complexed with an antibody FV fragment. Proc Natl Acad Sci U S A 94, 10547-10553 (1997).

59. A. M. Hartley et al., Structure of yeast cytochrome c oxidase in a supercomplex with cytochrome bc 1. Nature Structural and Molecular Biology 26, 78-83 (2019).

60. J. P. Hosler et al., Polar residues in helix VIII of subunit I of cytochrome c oxidase influence the activity and the structure of the active site. Biochemistry 35, 1077610783 (1996).

61. J. R. Fetter et al., Possible proton relay pathways in cytochrome c oxidase. Proc. Natl. Acad. Sci. USA 92, 1604-1608 (1995).

62. I. A. Smirnova, P. Ädelroth, R. B. Gennis, P. Brzezinski, Aspartate-132 in cytochrome c oxidase from Rhodobacter sphaeroides is involved in a two-step proton transfer during oxo-ferryl formation. Biochemistry 38, 6826-6833 (1999).

63. P. Brzezinski, A. L. Johansson, Variable proton-pumping stoichiometry in structural variants of cytochrome c oxidase. Biochimica et Biophysica Acta-Bioenergetics 1797, 710-723 (2010).

64. A. L. Johansson, M. Högbom, J. Carlsson, R. B. Gennis, P. Brzezinski, Role of aspartate 132 at the orifice of a proton pathway in cytochrome c oxidase. Proc. Natl. Acad. Sci. U. S. A. 110, 8912-8917 (2013). 
65. J. Berg, J. Liu, E. Svahn, S. Ferguson-Miller, P. Brzezinski, Structural changes at the surface of cytochrome $c$ oxidase alter the proton-pumping stoichiometry. Biochimica et Biophysica Acta-Bioenergetics 1861, 148116 (2020).

66. Y. Marantz, E. Nachliel, A. Aagaard, P. Brzezinski, M. Gutman, The proton collecting function of the inner surface of cytochrome $c$ oxidase from Rhodobacter sphaeroides. Proc Natl Acad Sci U S A 95, 8590-8595 (1998).

67. R. D. Sleator, C. Hill, Bacterial osmoadaptation: The role of osmolytes in bacterial stress and virulence. FEMS Microbiology Reviews 26, 49-71 (2002).

68. S. Jordan, M. I. Hutchings, T. Mascher, Cell envelope stress response in Gram-positive bacteria. FEMS Microbiology Reviews 32, 107-146 (2008).

69. H. Wennerström, E. V. Estrada, J. Danielsson, M. Oliveberg, Colloidal stability of the living cell. Proc. Natl. Acad. Sci. U. S. A. 117, 10113-10121 (2020).

70. H. B. Gray, J. R. Winkler, Long-range electron transfer. Proc. Natl. Acad. Sci. U. S. A. 102, 3534-3539 (2005).

71. S. Unthan et al., Beyond growth rate 0.6: What drives Corynebacterium glutamicum to higher growth rates in defined medium. Biotechnology and Bioengineering 111, 359-371 (2014).

72. A. Punjani, J. L. Rubinstein, D. J. Fleet, M. A. Brubaker, CryoSPARC: Algorithms for rapid unsupervised cryo-EM structure determination. Nature Methods 14, 290-296 (2017).

73. S. Q. Zheng et al., MotionCor2: anisotropic correction of beam-induced motion for improved cryo-electron microscopy. Nature Methods 14, 331-332 (2017).

74. J. L. Rubinstein, M. A. Brubaker, Alignment of cryo-EM movies of individual particles by optimization of image translations. Journal of Structural Biology 192, 188-195 (2015).

75. Schrodinger, LLC (2015) The PyMOL Molecular Graphics System, Version 1.8.

76. P. Emsley, B. Lohkamp, W. G. Scott, K. Cowtan, Features and development of Coot. Acta Crystallographica Section D: Biological Crystallography 66, 486-501 (2010).

77. S. F. Altschul, W. Gish, W. Miller, E. W. Myers, D. J. Lipman, Basic local alignment search tool. J. Mol. Biol. 215, 403-410 (1990).

78. P. V. Afonine et al., Real-space refinement in PHENIX for cryo-EM and crystallography. Acta Crystallographica Section D: Structural Biology 74, 531-544 (2018).

\section{Figure legends}

Figure 1 Reactions catalyzed by $\mathrm{CIII}$ and $\mathrm{CIV}$. Upon binding of $\mathrm{QH}_{2}$ at the $\mathrm{Q}_{\mathrm{P}}$ site in $\mathrm{CIIl}$ one electron is transferred to $\mathrm{FeS}$, along the $\mathrm{C}$ branch, and one along the $\mathrm{B}$ branch, via hemes $b_{\mathrm{L}}$ and $b_{\mathrm{H}}$ to the $\mathrm{Q}$ at the $\mathrm{Q}_{N}$ site. The reaction sequence is repeated upon binding of a second $\mathrm{QH}_{2}$ at the $\mathrm{Q}_{\mathrm{P}}$ site. In canonical CIII the mobile FeS ectodomain moves from the electron-receiving B position to the electron-donating $\mathrm{C}$ position to transfer an electron to $c y t . c_{1}$, which reduced water-soluble cyt. $c$. Oxidation of $\mathrm{QH}_{2}$ at the $\mathrm{Q}_{\mathrm{P}}$ site is associated with proton release to the $p$ side of the membrane while reduction of $Q$ at the $Q_{N}$ site is associated with proton uptake from the $n$ side. Reduced cyt. $c$ transfers electrons to $\mathrm{Cu}_{A}$, heme $a$, and the heme $a_{3} / \mathrm{Cu}_{B}$ catalytic site of CIV where $\mathrm{O}_{2}$ is reduced to $\mathrm{H}_{2} \mathrm{O}$. Each electron transfer from cyt. $c$ to the catalytic site is linked to pumping of one 
proton across the membrane. The lower part of the figure shows the M. smegmatis $\mathrm{II}_{2} \mathrm{IV} \mathrm{V}_{2}$ supercomplex where electrons from CIII to CIV are transferred via a di-heme cyt. cc domain.

Figure 2 Structure of the supercomplex. (A) The entire $\mathrm{III}_{2} I \mathrm{~V}_{2}$ supercomplex with core subunits in light $\left(\mathrm{CIII}_{2}\right)$ or dark (CIV) grey, and accessory subunits in color as indicated. (B) The core of complex IV with $\mathrm{CtaD}, \mathrm{CtaC}, \mathrm{CtaE}$ and $\mathrm{CtaF}$, shown in color and a surface representation of the entire CIV in dark grey. (C) The complex $\mathrm{III}_{2}$ dimer in which each monomer is composed of subunits QcrA-C. The right-hand side monomer is shown in grey while the subunits of the lefthand side monomer are colored. Note that TMH 1 of QcrA is integrated into the other half of the dimer. A surface representation of the entire $\mathrm{ClII}_{2}$ is shown in light grey.

Figure 3 Q-binding sites on the periplasmic side. (A) Density modeled as an MQ in the $Q_{p}$ site, MQ1a. Protonatable residues discussed in the text and the PDVY motif in the QcrB subunit are shown. Residue H355 is found in subunit QcrA. (B) The $Q_{P}$ site with bound MQ1a. (C) Position of MQ1a in relation to the FeS cluster and heme $b_{\mathrm{L}}$, as well as a second MQ in site MQ2. In the inset the approximate position of this area in one CIII monomer of the supercomplex is marked with a red circle. (D,E) Amino-acid residues that define the MQ2 binding site. The MQ2 density is shown in panel D.

Figure 4 The Q-cycle electron bifurcation in C. glutamicum CIII. The first electron and proton from $\mathrm{MQH}_{2}$ are transferred to FeS and His355, respectively. The second electron and proton are transferred to heme $b_{\mathrm{L}}$ and Asp302, respectively. After this charge separation electron transfer from FeS (to heme $c$, not shwon) is not possible because His355 cannot be deprotonated until the proton is released from Asp302. This sequence of events ensures that the second electron is transferred along the $\mathrm{B}$ branch before the first electron is transferred along the $\mathrm{C}$ branch.

Figure 5 The $C$. glutamicum supercomplex. (A) The right- and left-hand side halves of the supercomplex show the reactions catalyzed by CIII and CIV, and distances between the cofactors, respectively. Residues of the $\mathrm{D}$ and $\mathrm{K}$ pathways in CIV are labelled in the left-hand side. (B) The entry point of the $D$ pathway in complex IV. Subunits that contribute residues that define the entry point of the D pathway of CIV are shown in color. A complex III QcrB loop (green) forms a "lid" over the D-pathway entry point cavity. This "lid" harbors a number of protonatable residues that define a proton pathway leading from the cytoplasmic side to the $D$ pathway entry point defined by Asp116. 


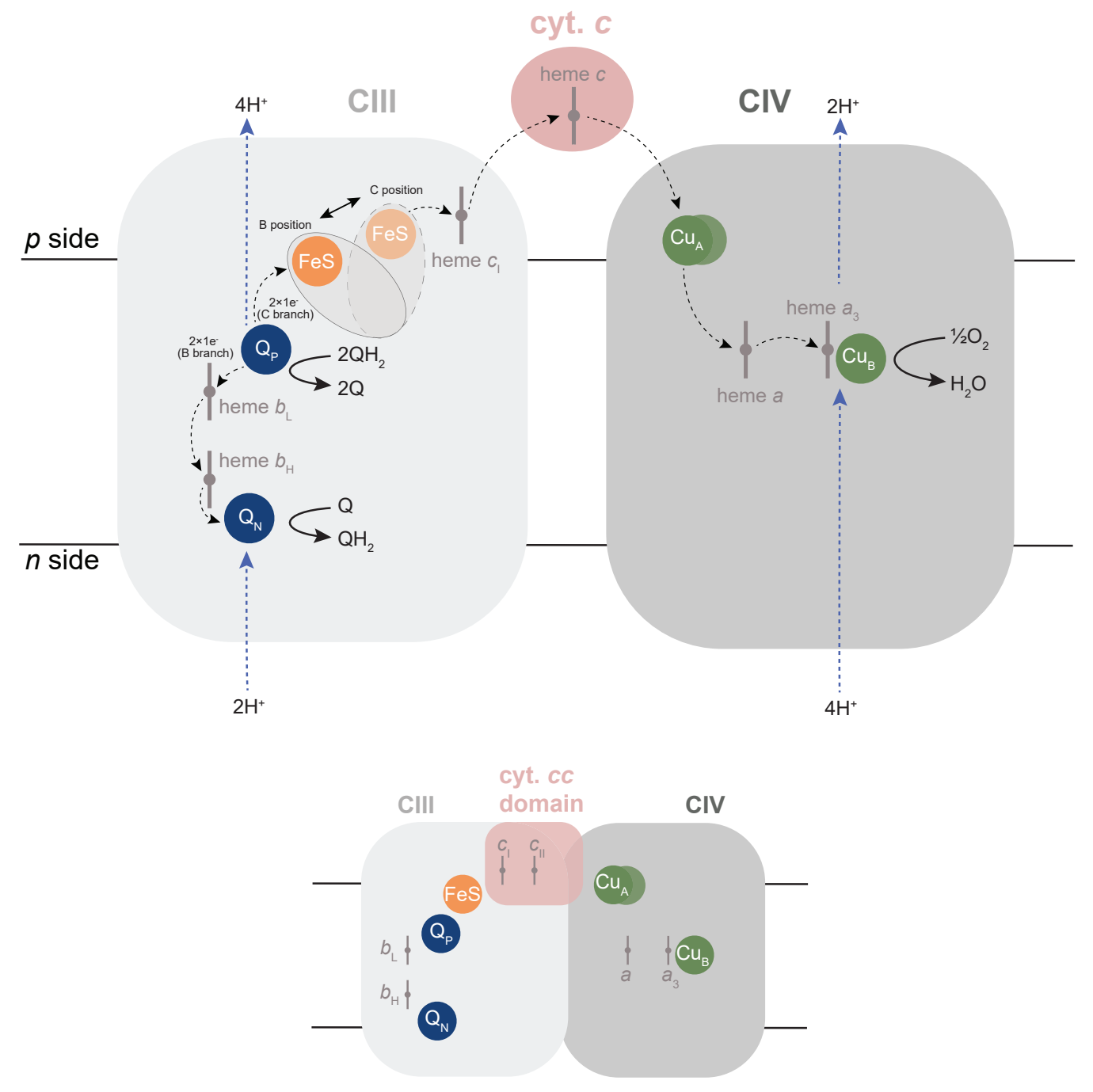


A
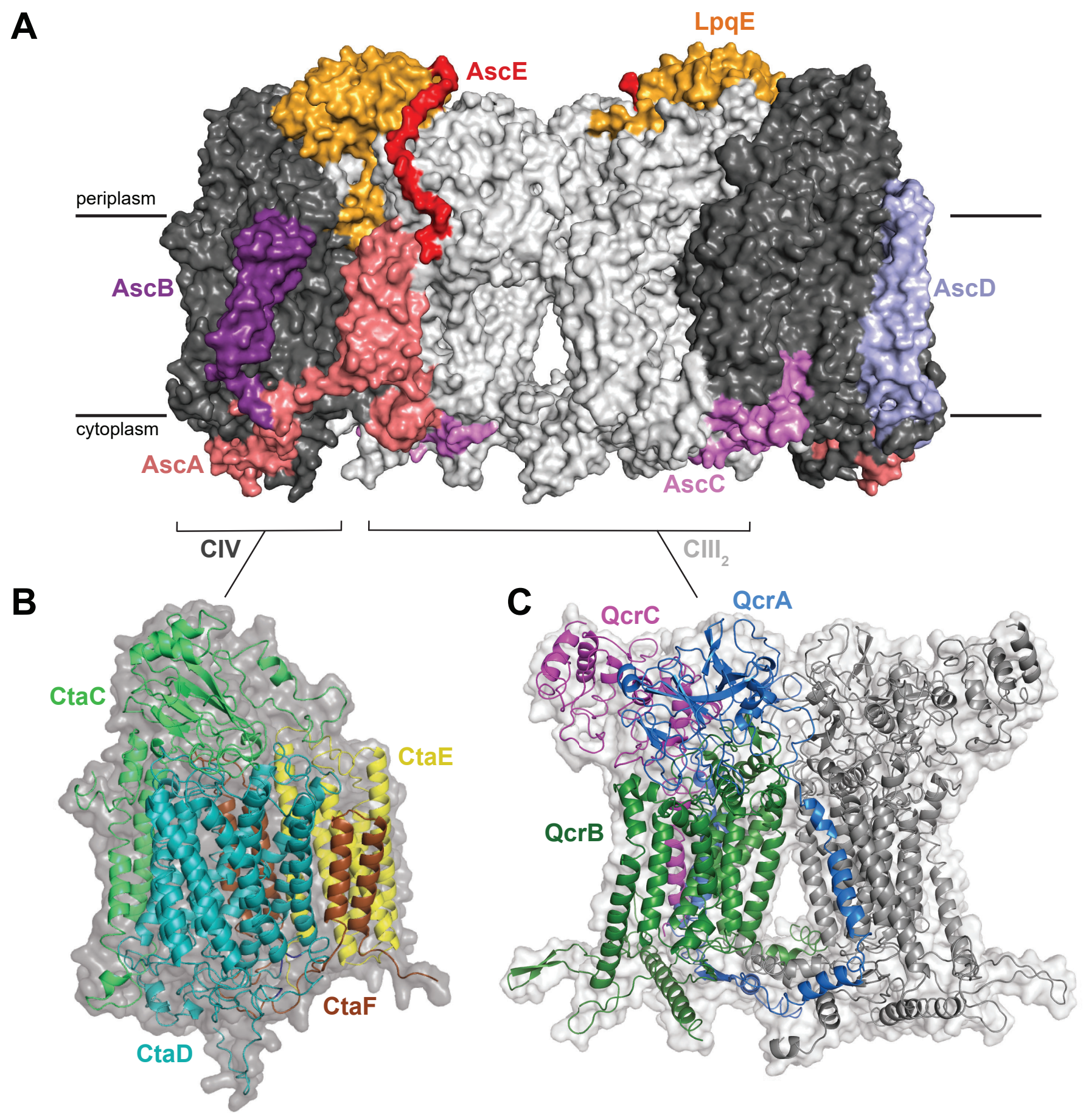


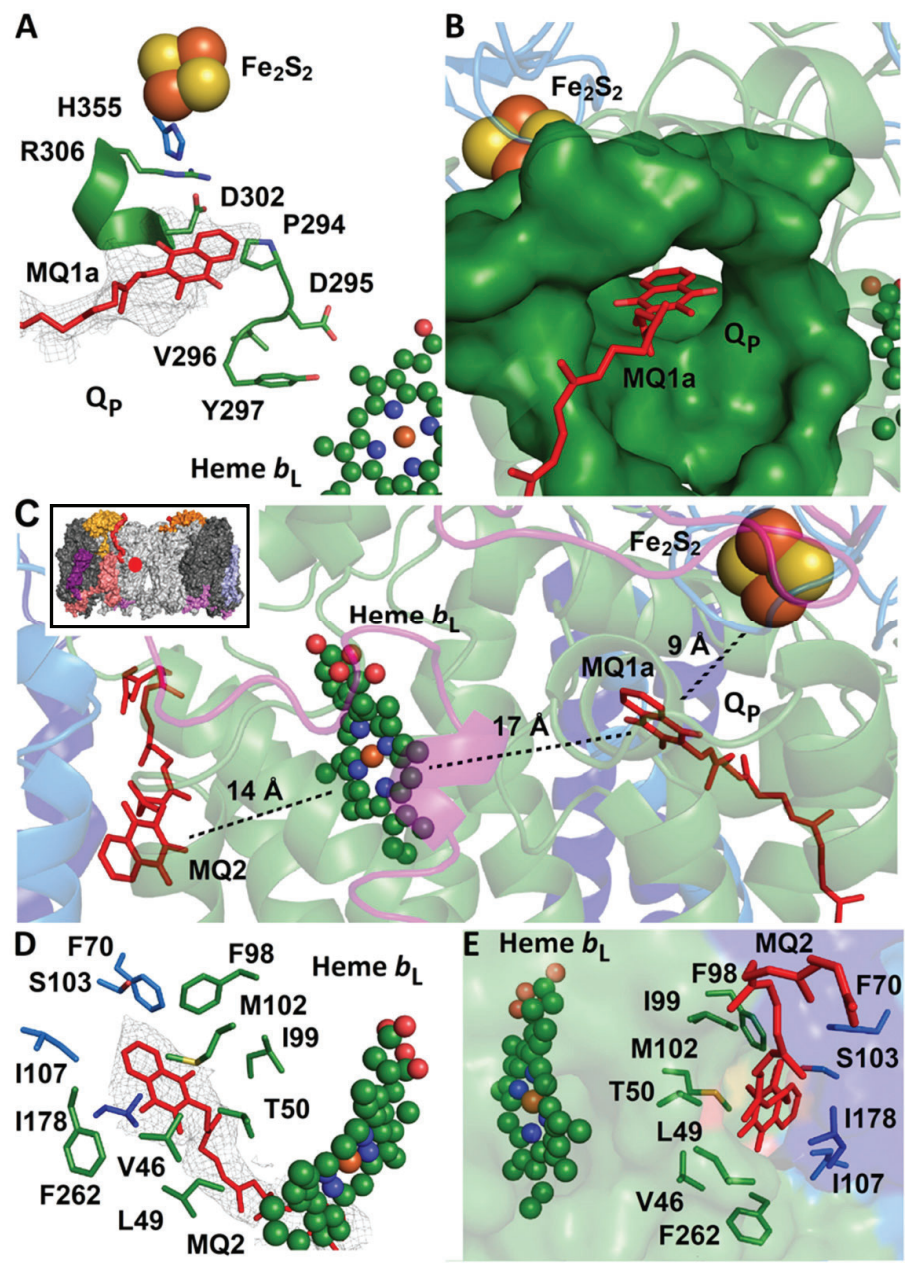




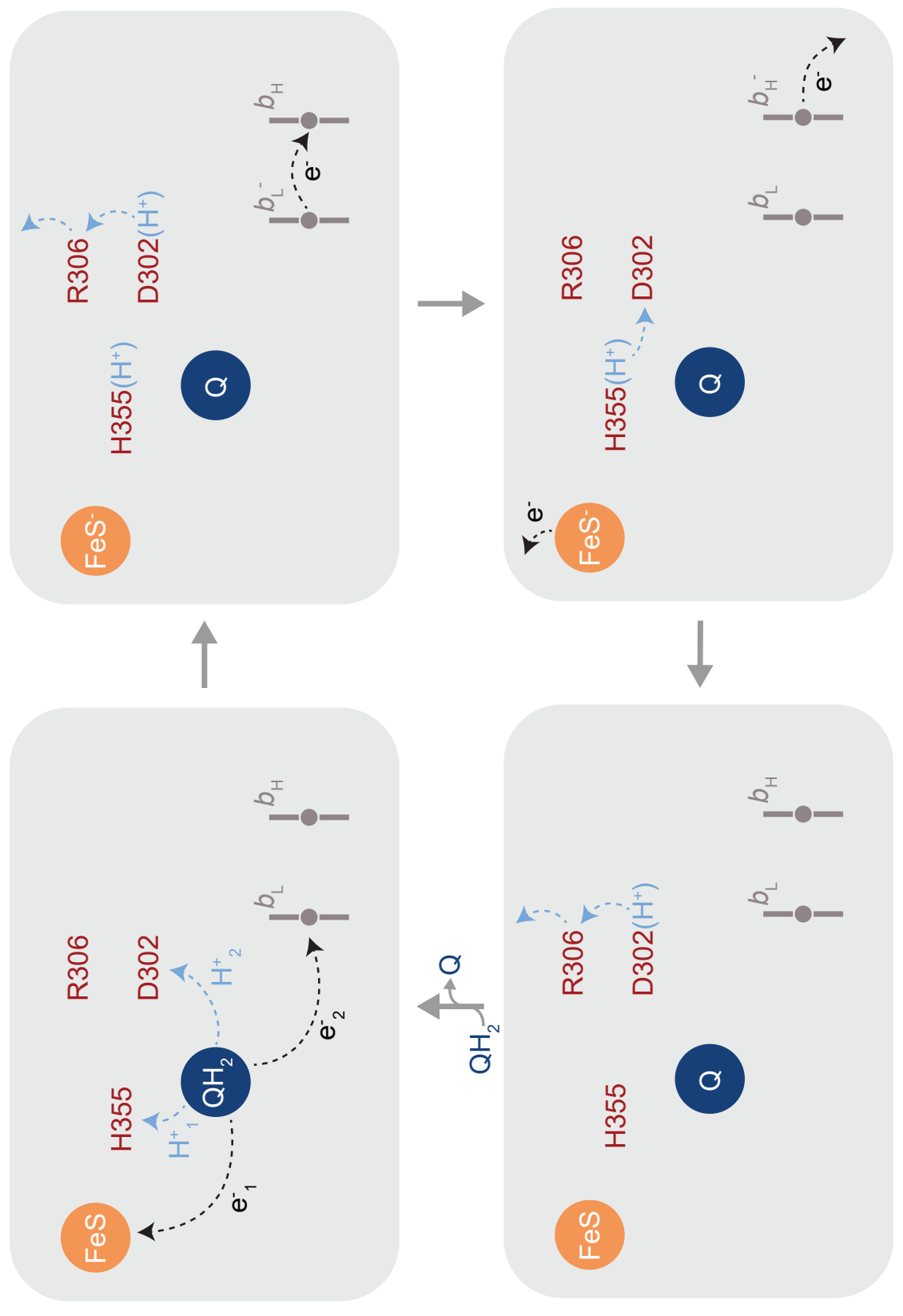



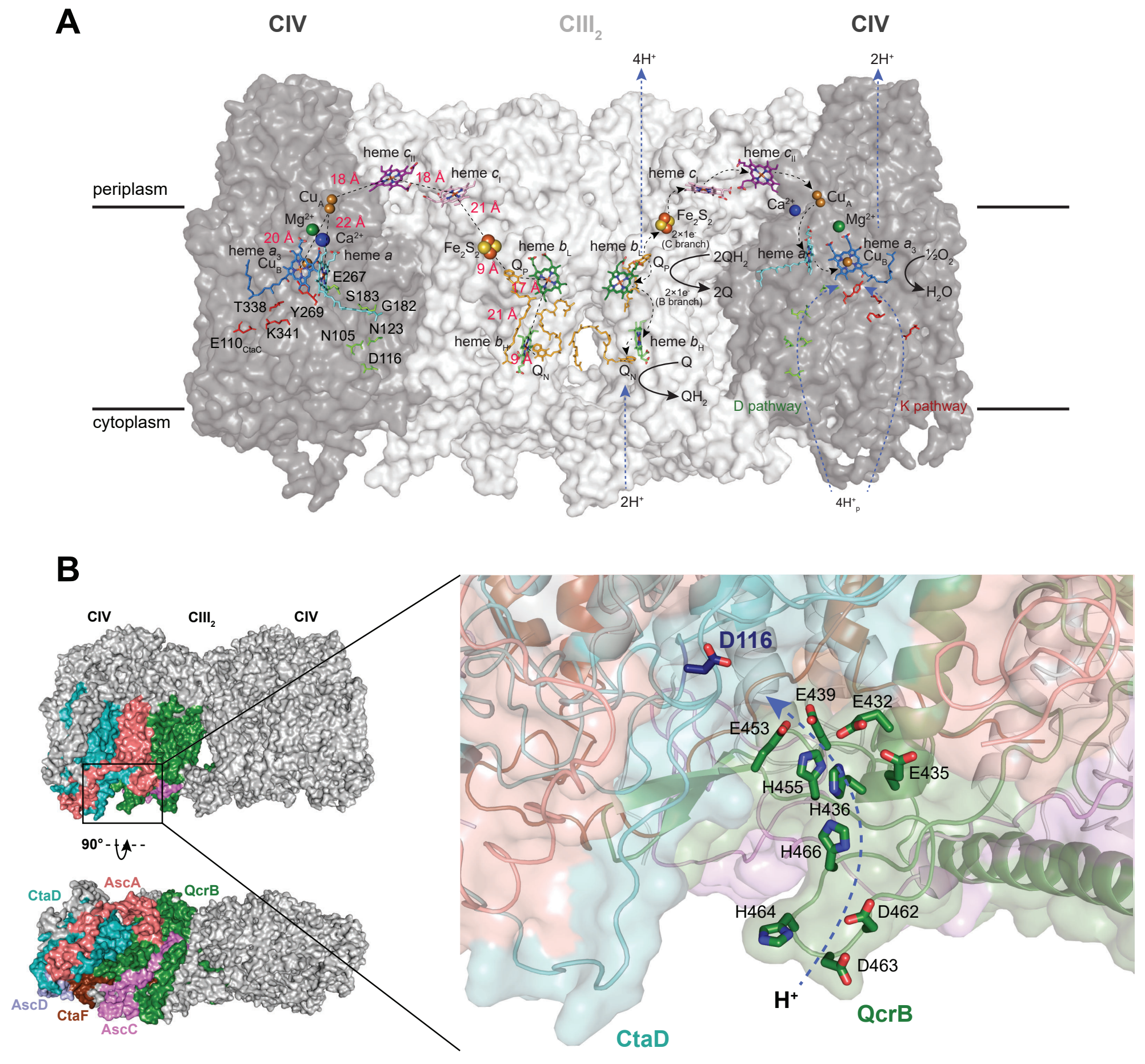